\title{
WEB-BASED INSTRUCTION AND ITS IMPACT ON THE LEARNING ACTIVITY OF MEDICAL STUDENTS: A REVIEW
}

\author{
Jarmila Potomkova ${ }^{\mathrm{a}}$, Vladimir Mihal ${ }^{\mathrm{b}}$, Cestmir Cihalik ${ }^{\mathrm{c}}$
}

\author{
a Medical Learning Resource Centre, Palacky University Library, 77503 Olomouc, Czech Republic. \\ b Department of Pediatrics, Faculty of Medicine and Dentistry, Palacky University, 775 20 Olomouc, Czech Republic \\ c Department of Internal Medicine, T. Bata Regional Hospital, 76275 Zlín, Czech Republic \\ e-mail:potomkov@tunw.upol.cz
}

Received: September 20, 2006; November 1, 2006

Key words: Computer-assisted instruction/Medical education/Web-based tutorials/Self-directed learning

Background: The aim of this review was to summarise the experience on implementation of information technology to support the teaching and learning process in medicine. Particular attention was paid to web-based tutorials, their impact on increasing the effectiveness of medical instruction and motivation of students towards self-directed learning. Most of the studies selected for the purpose of the review comprised evaluation of the web-tutorials in view of practical implementation, strengths, weaknesses, and main preferences in comparison with traditional lecture-based education.

Method and results: We searched MEDLINE via PubMed using MeSH term "computer-assisted learning" between 1996 and 2005 and selected for inclusion in this review were studies on the implementation and evaluation of web based tutorials in medical education. Additional related papers were obtained through cross-referencing. We found that overall, students prefer Web tutorials to traditional lecture-based classes for accessibility, ease of use, freedom of navigation, high medical image quality and advantage of repeated practice, that web-based learning has been continually developing and that it is a very important tool in Evidence Based Medicine.

Conclusions: Web based education is an important tool in medical training. It will require transformation in the way medicine is taught from instructor based to self directed learning. It is above all seen as a device for information retrieval and storage.

\section{INTRODUCTION}

Large educational establishments are responsible for facilitating the uptake, development and implementation of technology in teaching and learning. Development of new learning environments can promote active participation through repeated practice, encourage shared experiences and enhance student motivation ${ }^{1}$. The World Wide Web is now a rich educational resource that promotes and facilitates student learning and is increasingly being used to deliver course content in medical and nursing programs. It has evolved from computer-assisted instruction that started approximately 30 years ago ${ }^{2}$.

In 1995, McEnery et al. $^{3}$ recognised the potential of utilizing the world wide web as a method for interactive medical instruction for geographically dispersed students. In medicine in particular, the integration of Web-based instruction into medical curricula is a great challenge both for faculty and students to use the technology efficiently and effectively to support teaching and learning.

Teaching Web-based courses are a new experience for most university teachers and require adoption of new concepts of role of faculty. Issues to be considered by faculty when introducing Web-based instruction include instructional design, faculty-student interactions, time and technology management skills and student outcome evaluation. Students preferring faculty-directed classroom learning will also have to consider their roles in the process of redesigning faculty-directed classroom learning for Web-based instruction ${ }^{4}$.

As far as the terminology is concerned, Daniel ${ }^{5}$ suggested that a more appropriate term for web-based education would be web-enhanced education, since the use of technology augments the learning potential of each individual course.

\section{METHODS OF REVIEW}

We searched MEDLINE via PubMed using MeSH term "computer-assisted learning" between 1996 and 2005. The studies dealing with implementation and evaluation of web-based tutorials in medical education were selected for inclusion in the review. Additional papers on general principles of self-directed computer-assisted instruction were obtained through cross-referencing. 


\section{RESULTS}

\section{From Lecture-Based to Computer-Assisted Teaching and Learning}

Yolton and deCalesta ${ }^{6}$ foresaw a revolution in education that would involve a shift from the traditional lecture style of information presentation toward a more active style of learning. Many educators now believe that students must actively participate in the learning process so that information be truly understood, retained and transformed into knowledge. This revolution has led to an increased understanding that the mass of knowledge in most professions is too large to be absorbed. These concepts have triggered innovative, computer-based educational techniques.

Based on their 30 years of experience with computeraided pharmacology instruction, Kerecsen and Pazdernik ${ }^{2}$ developed a computer-assisted teaching system comprising both computer-assisted instruction (CAI) and computer-managed instruction (CMI). This system evolved from microprocessors to the current World Wide Web system. It was first used in the early 1970s to teach pharmacology to second year medical students. CAI consists of educational programs and the majority of them are self-instructional requiring fill-in-the-blank or response to simple multiple-choice questions. Initially, they were written by faculty members, later many of the programs were elaborated by medical students in cooperation with faculty members who were experts in particular fields.

In their experimental study Foust, Tannery and Detlefsen $^{7}$ focused on Web-based tutorial implementation for the delivery of health sciences libary user education that is one of the numerous responsibilities of reference medical librarians. The experimental study was undertaken to determine if a trial web-based tutorial could be used as an effective alternative to the traditional library lecture. The authors emphasized the significance of evaluating web-based tutorials as a method of learning, indicating that they could serve as an alternative to the more traditional lecture delivery. This finding is extremely important to those who plan library training programs and information management education. It may be concluded that the web-based tutorials can do at least as well as a traditional classroom style lecture, and consequently the delivery of web-based instruction can become a substitution form of user education.

Perlman, Weston, and Gisel ${ }^{1}$ reported the importance of web-based learning environments offering interactive, authentic, self-directed learning opportunities in occupational therapy educational programs. They have claimed that Web-based technology represents a new instructional mehod to support cognitive learning, including the analysis, synthesis and evaluation of knowledge. The form of a case-based tutorial gives opportunitites to practice and receive feedback. These authors also describe the theoretical foundations of tutorial development, implementation and evaluation. They give a practical outline of tutorial constructs in terms of setting, user-friendly menu, interaction with the hyper-textual environment and content-re- lated formats with a special interest in the value of various types of questions facilitating final exams.

Similarly, Kemp, Morrison, and Ross ${ }^{8}$ stressed the need for instructors who are also the designers of tutorials to propose student-directed instruction taking student characteristics and learning styles into account. It was decided that the instruction should be seen through the perspective of the learner rather than the content. Compared to traditional lecture-based teaching, instructional strategies need to change not only what is taught, but how it is learned ${ }^{9}$.

\section{Effectiveness of Web-Based Instruction in Medicine}

Even if a large numer of studies have described comparable outcomes from Web-based and traditional classroom instruction, there are few such studies for medical curricula.

Gallagher et al. ${ }^{10}$ examined the effectiveness of alternative methods of course delivery by comparing student profiles and instructional outcomes from a dental hygiene gerontology course offered both on the Web and in a traditional classroom setting. Questionnaires were sent to both groups of students completing the course. The data collected included familiarity with Web-based instruction, extent of prior computer training, and student evaluations of course effectiveness. A comparison of student characteristics participating in the two course formats revealed statistically significant differences in instructional outcomes. Student retention of course material six months after completion of the course was greater in the Webbased format. Students selecting a Web-based course format demonstrated greater motivation and learning success based on final course grades, completion of assignments, and knowledge retention over time. Age, previous experience with online courses, and selection of teaching mode may have been confounding factors however.

Yolton and deCalesta ${ }^{6}$ examined a variety of Webbased educational methodologies in optometry classes, ranging from shifting classes totally to the Web, to using the Web for pre-class preparation. Initial student acceptance of Web-based courses was good, but an undesirable trend emerged as a tendency for students to print out Webpresented material rather than reading from the computer screen. Other concerns with shifting material from lecture to the Web include peer and administrator acceptance of this teaching style and issues associated with valuation of professors who no longer give stand-up lectures.

Green at al. ${ }^{11}$ emphasized the role of web tutorials produced by health sciences libraries to provide a service in new electronic resources to users outside the library and around the world. Online tutorials are a valuable means of providing instruction on how to access and use important resources. The authors described among others how the work could be evaluated.

Another evaluation study ${ }^{12}$ focused on student satisfaction with a mixed learning method for teaching radiologic anatomy that integrated web-based instruction with small group and didactic teaching. The mixed learning model comprised a brief didactic introduction followed by web- 
based structured learning modules including self-study cases prior to the lab instruction, follow-up cases, and twice-weekly optional review sessions. Students and lab instructors were assessed on content and design. All the radiology labs were rated "very useful" or "useful".

Buckley and Toto ${ }^{13}$ made an attempt to discover how students learned in an online learning environment as well as to investigate how online communication patterns differed from those in a face-to-face class. In their evaluation study the authors used students participating in a graduate program in instructional technology. Various activities and assignments were used to procure data on student attitudes toward an online learning environment. Over a period of 3 semesters, student data were collected using qualitative and quantitative methods, including observation, surveys, tests, in-class discussions, and e-mail communication analysis. The results of the study confirmed the importance of regular assessment of tutorial quality.

Johnson, Xing and Yang ${ }^{14}$ accentuated the main features of hypertutorials, such as presentation, learner control, practice, feedback, and elaborative learning resources based on the results of a randomized controlled trial. They showed that the source of hypertutorial superiority in student evaluations of health informatics instruction lay in their hypertutorial features. Randomized comparisons between the two versions containing the same text and graphics differed in the presence or absence of 4 tutorial features: elaborative learning resources, practice, feedback, and amount of learner control. Students gave high evaluations to both Web-based methodologies, but consistently rated the hypertutorial lessons as superior.

In the same year, Erickson et al. ${ }^{15}$ published the results of a randomized controlled trial to compare pharmacy students' acquired knowledge and technical skills after a traditional lecture versus a Web-based tutorial. There was no significant difference in the baseline knowledge test scores between the groups. The pharmacy students confirmed that the Web-based tutorial was as effective as the standard lecture format. The authors stressed the necessity of further testing to assess the longitudinal effect of the program.

Meyer ${ }^{16}$ summarized the promising results of an evaluation study focused on the quality of his software developed as a tutorial to support nursing education and training dispensation. Student feedback was useful and suggestions made by the participants who completed the questionnaire were used to improve and modify the product.

Boyle and Wambach ${ }^{17}$ verified a conceptual model for graduate nursing Web-based instruction in which learner-centered pedagogic philosophies and interaction techniques were combined to produce active learning at each phase of the Web-based courses. The focus was on asynchronous techniques as the dominant form of communication.

In a modified cross-over design Ludlow and Platin ${ }^{18}$ compared self-guided slide/tape (ST) and web page (WP) instruction in normal radiographic anatomy using objective test performance and subjective preference of first year dental students. The two groups of students switched presentation technologies to learn anatomy in panoramic images. Students completed on-line and conventional quizzes covering WP'and ST materials, as well as a voluntary survey. A total of 71 per cent of students preferred WP for accessibility, ease of use, freedom of navigation, and image quality $(\mathrm{p}<.01)$.

According to Thiele, Allen and Stucky ${ }^{19}$ web-based instruction requires transformation from traditional teaching strategies to directed independent learning. Much attention must be given to the creation of effective interactive learning experiences that support student-to-student and student-to-faculty interactions. Evaluation must be done of how content is learned, the effects of the use of computers on learning, and perceived barriers to learning. Students' responses to Web-based education should also be considered. The authors analyzed the responses of 58 undergraduate and 13 graduate students to their initial experiences with Web-based instruction (WBI). While the educational levels of the students and the courses in which the transition to WBI took place differed for the two groups, their computer expertise did not, and similar responses to Web-based learning were noted. Differences in the types of courses and the expertise of the two faculties with regard to WBI also contributed to the production of some dissimilar responses.

Koch and Gobell ${ }^{20}$ evaluated the effectiveness of an online tutorial for research design and statistics in psychology. Initial research suggested that students using the tutorial were more accurate in their decisions. Students were also more confident in the decisions and found them easier to make when using the tutorial. The practice with the tutorial improved problem-solving ability in subsequent design and statistics scenarios.

\section{Implictions for Practice: Strengths and Weaknesses}

Although the use of technology in teaching and learning is becoming more prevalent, we have to take into consideration strengths and weaknesses. It may become a beneficial investment if the strategy is supported and well balanced with all other instructional components, matches the learning outcomes, supports self-directed learning and enables practice and feedback. As a result, learners will be able to acquire new knowledge, manipulate and change it through interactivity and enrich it through feedback ${ }^{1}$.

In an extensive study Kerecsen and Pazdernik ${ }^{2}$ discovered that students preferred more linear paradigm (text - question - response - test - question -response - text) in a pharmacology web tutorial. With the more branching format the student might select the wrong answer to get more information. Students found the „Drill and Practice“ programs the most time-efficient way of mastering exam material. The success of computer-assisted programs was found to depend on their content rather than sophisticated programs or technology. Computers are also very valuable as search engines for obtaining the most important information in a specific field. Evidence based medicine (EBM) has become very important in the training of individuals in clinical rotations and residency programs. Therefore, it is important that in the first 2 years of un- 
degraduate medical education students are trained to be able to use computers to develop problem-solving skills as well as to search for the most appropriate information for using EBM principles. The biggest challenge is the constant requirement to update wrt both content and changing technologies.

Mbarika et al. ${ }^{21}$ confirmed that learners had a stronger sense of efficacy and perceived skill development when using multimedia versus paper-based tasks. These attributes are due to the learning-driven constructs within the technology which can be described as challenging, interesting, reflective, and interactive while learning with others. The multimedia approach is successful in providing situated learning in the classroom, in teaching difficult concepts, and in transferring theory into practice. It is not the multimedia itself, but the learning opportunities and instructional strategies that the medium affords.

Hung and Wong ${ }^{22}$ observed that technology could supplement classroom and lab instruction and mirror the content taught in these situations. It has the advantage of allowing repeated practice. The learner continually engages in a process of active learning.

Similarly, Duchaste ${ }^{23}$ emphasized the importance of building learner confidence through successful completion of a learning task. If the learners feel challenged, they may gain interest and motivation which leads to repeated practice and further conceptual and skill development. Nevertheless, it would not be wise to exaggerate the significance of information technology, because students actually do not learn from technologies, but with technologies ${ }^{24}$. Extensive observations ${ }^{1}$ have shown that self-instruction by students can produce feedback to the instructors and this can impact further development. Feedback can also be gathered from asynchronous and synchronous on-line discussions between students and students and instructors. All these responses reveal the strengths and weaknesses of the tutorial and enable the instructors to reflect, review and revise. The authors ${ }^{1}$ collected valuable data to help identify positive trends in the instruction, and flaws and deficiencies which could be corrected immediately as well as to determine the initial impact on student learning. Long-term results led to the conclusion that learning could be definitely improved as a result of the added practice, feedback, and repetition. This had been expected in particular due to the increased opportunity to practice and receive feedback. These results clearly demonstrate that the technology is a powerful instructional tool for opportunity to practice and receive feedback. This represents an important contribution to increasing practical application of skills as well as cognitive learning. The tutorials also offer immediate feedback to the student through comparison of answers leading to increased motivation to learn. It is important to maintain a counterbalance between the methods and the level and domains of learning and evaluation. Students may be more familiar with technology from year to year; students grow with it. It may be useful to gather information on how many students implement the tutorial alone or in groups of two and three, and to investigate the influence of collaborattive implementation of learning. In contrast, some students report difficulty when navigating through the hyperlinks and when sending results electronically to instructors. It is inevitable to monitor the function of all hyperlinks and to provide regular assistance toward troubleshooting. This requires consistent reviewing of the accessibility and interface design of the tutorial in collabortion with the technology experts

As confirmed by Foust, Tannery and Detlefsen ${ }^{7}$, tutorials enable active learning, reduced student memorization, and increased use of computers as information storage and retrieval devices, which could change fundamentally the way in which future doctors, patients, and computers interact in clinical settings.

An undesirable trend was observed by Yolton and deCalesta ${ }^{6}$ who recorded the tendency for students to print out Web-presented material rather than reading from the computer screen.

\section{CONCLUSIONS}

Most of the studies analyzed for the purpose of this review point out strengths rather than weaknesses of webbased instruction used in medical education as an innovative tool for increasing effectiveness of both teaching and learning

It may be concluded that computer-assisted instruction since its beginnings more than 30 years ago has been undergoing dynamic development towards Web-based education. In medicine, web tutorials represent an effective educational tool supporting self-directed learning. Students prefer Web tutorials to traditional lecture-based classes for their accessibility, ease of use, freedom of navigation, high medical image quality and an advantage of repeated practice.

All of these aspects lead to increased motivation to acquire comprehensive medical knowledge and master clinical skills necessary for practising evidence based medicine. It should be taken into consideration that tutorial design and maintenance remains dependent on the expertise of programmers and instructional design consultants. This diminishes spontaneity for revision and corrections that may delay effective implementation.

Last but not least, it also requires funding to pay for such service. From the methodological point of view, Web-based instruction requires transformation from traditional teaching strategies to directed independent learning. Ragular evaluation must be carried out of how content is learned, the effects of the use of computers on learning, and perceived barriers to learning.

\section{ACKNOWLEDGEMENTS}

This paper is related to the developmental programme of Palacky University Faculty of Medicine and Dentistry (Olomouc, Czech Republic), supported by the Ministry of Education, Youth and Physical Culture of the Czech 
Republic. The web-tutorial under construction is available at http//noe.upol.cz.

\section{REFERENCES}

1. Perlman C, Weston C, Gisel E. A web based tutorial to enhance student learning of activity analysis. Can J Occup Thera 2005; 72(3):153-163.

2. Kerecsen L, Pazdernik TL. From mainframe to Web-nased: 30 years of experience in computer-aided instruction of pharmacology Naunyn-Schmiideberg's Arch Pharmacol, 2002; DOI 10.1007/ s00210-002-0558-8.

3. McEnery KW et al. A method for interactive medical instruction utilizing the World Wide Web. Proc Annu Symp Comput Appl Med Care 1995; 502-7.

4. Halstead JA, Coudret NA. Implementing Web-based instruction in a school of nursing: implications for faculty and students. J Prof Nurs. 2000 Sep-Oct; 16(5):273-81

5. Daniel J Sir. The university of the future and the future of universities. Keynote address presented at the $25^{\text {th }}$ Internationa Conference: Improving university learning and teaching. Frankfurt, Germany; 2000.

6. Yolton RL, deCalesta D. Pacific's experience with Web-based instruction: bats in the belfry or Webs in the classrom? Optometry 2000; 71(1):20-28.

7. Foust JE, Tannery NH, Detlefsen EG. Implementation of a Webbased tutorial. Bull Med Libr Assoc, 1999; 87(4):477-479.

8. Kemp IE, Morrison GR, Ross SM. Designing effective instruction. $19992^{\text {nd }}$ ed. New York, NY: Wiley and Sons.

9. Laurillard D. Rethinking university teaching: A framework for the effective use of educational technology.1993. New York: Routledge.

10. Gallagher JE et al. Web-based vs. traditional classroom instruction in gerontology: a pilot study.J Dent Hyg. 2005; 79(3):7.

11. Green BF at al. Web tutorials bibliographic instruction in a new medium. Med Ref Serv Q 2005; 25(1):83-91.
12. Shaffer K, Small JE. Blended learning in medical education: use of an integrated approach with web-based small group modules and didactic instruction for teaching radiologic anatomy. Acad Radiol. 2004; 11(9):1059-70.

13. Buckley J, Toto R. Assessment techniques for web-based instruction: lessons learned from teaching a graduate course in instructional technology. Semin Perioper Nurs. 2001; 10(2):97-103.

14. Johnson CW, Xing Y, Yang L. Randomized comparisons among health informatics students identify hypertutorial features as improving web-based instruction. AMIA Ann Symp Proc 2003; 328-30.

15. Erickson SR et al. Lecture versus Web tutorial for pharmacy students' learning of MDI technique. Ann Pharmacother 2003; 37(4):500-5.

16. Meyer SM. A formative evaluation of a Web-based tutorial. Curationis 2001; 24(3):12-8.

17. Boyle DK, Wambach KA. Interaction in graduate nursing Webbased instruction. J Prof Nurs 2001; 17(3):128-34.

18. Ludlow JB, Platin E. A comparison of Web page and slide/tape for instruction in periapical and panoramic radiographic anatomy. $\mathrm{J}$ Dent Educ. 2000 Apr; 64(4):269-75.

19. Thiele JE, Allen C, Stucky M. Effects of Web-based instruction on learning behaviors of undergraduate and graduate students. Nurs \& Health Care Persp 1999; 20(4):199-203.

20. Koch C, Gobell J. A hypertext-based tutorial with links to the Web for teaching statistics and research methods. Behav Res Meth Instrum Comput 1999; 31(1):7-13.

21. Mbarika VW et al. Importance of learning-driven constructs of perceived skill development when using multimedia instructional materials. J Educ Technol Syst 2000-2001; 29:67-87.

22. Hung DWL, Wong PSK. Toward an information and instructional technology research framework for learning and instruction. Educat Technol 2000; 40:61-62.

23. Duchastel P. A web-based model for university instruction. J Educ Technol Syst 1996-1997; 25:221-228.

24. Jonassen DH. Transforming learning with technology: beyond modernism and post- modernism or whoever controls the technology creates the reality. Educat Technol 2000; 40:21-25. 